\title{
Bacterial biofilm in children with chronic rhinosinusitis and chronic adenoiditis
}

\author{
Radmila Anca Bugari ${ }^{1,2}$, Sorin Baschir ${ }^{1,3}$, Ciprian Mihali ${ }^{4,5}$, Luminita Turcin ${ }^{6}$, \\ Dana Simona Chita ${ }^{7}$, Adrian Cosmin $\mathrm{Ilie}^{8}$, Alexandru Chioreanu', Afilon Jompan ${ }^{6}$ \\ ${ }^{1}$ Otorhinolaryngology, "Vasile Goldis" Western University, Arad, Romania \\ 2Otorhinolaryngology, "Dr. Turcin" Clinic, Arad, Romania \\ ${ }^{3}$ Otorhinolaryngology, County Clinical Hospital, Arad, Romania \\ ${ }^{4}$ Cell and Molecular Biology, Electron Microscopy, "Vasile Goldis" Western University, Arad, Romania \\ ${ }^{5}$ Molecular Biology Laboratory Research and Development Station for Bovine, Arad, Romania \\ ${ }^{6}$ General Medicine, "Vasile Goldis" Western University, Arad, Romania \\ ${ }^{7}$ Neurology, "Vasile Goldis" Western University, Arad, Romania \\ ${ }^{8}$ Public Health and Sanitary Management, "Victor Babes" University of Medicine and Pharmacy, \\ Timisoara, Romania
}

\begin{abstract}
Chronic rhinosinusitis with chronic adenoiditis in children represents a global public health issue, seriously affecting the quality of parents and children life, because of its irritating symptoms like intermittent snoring, mouth breathing, dry mouth, nasal obstruction, headaches increased irritability and focus disorders on children.

Bacterial biofilms are highly associated with the chronic infectious processes in children. Correct therapeutical management of this diagnostic combination is mandatory to improve the quality of one's life.

Objectives. The aim of the study is: to observe the ratio of adenoid mucosa covered with bacterial biofilm extracted from the nasopharynx of 50 paediatric patients suffering of chronic rhinosinusitis (RSC) and chronic adenoiditis (CA); and to point the fact that the adenoids contaminated with bacterial biofilm are a generator for chronic upper airway infections in children.

Material and methods. We have measured using an image analysis program the bacterial biofilm covering the entire surface of the extracted adenoids mases, from 28 girls and 22 boys aged between 5 and 12 years diagnosed with CRS and CA.

Control visits were performed to verify symptom improvement at 1,3 and 6 months.

Outcomes. Adenoids extracted from paediatric patients diagnosed with CRS and CA presented bacterial biofilms coverage on almost the entire mucosa $(86.75 \%)$.

Conclusions. Adenoid mases removed from paediatric patients with CSR and CA have most of their mucosal covered with bacterial biofilm. In the nasopharynx of paediatric patients with CSR and CA, bacterial biofilm can play the role of a constant fountain of infection. Adenoid mass removal explains the symptomatic improvement observed post operatory in the CRS with CA paediatric patients that do not respond to antibiotic therapy.
\end{abstract}

Keywords: scanning electron microscopy, chronic adenoiditis, chronic rhinosinusitis, bacterial biofilm

\section{INTRODUCTION}

Chronic rhinosinusitis (CRS) with chronic adenoiditis (CA) in paediatric patients represents a constant issue, with a great impact on the economy and on the quality of life. Chronic rhinosinusitis is more common in the paediatric population due to an increased frequency of exposure to upper respiratory tract infections in this group of patients.
The first-time bacterial biofilm was described in medical literature, was by Antonie van Leeuwenhoek (1632-1723) observing the dental plaque at the microscope [1].

The evolution of the bacteria as part of a biofilm presents 3 stages: stage 1 - the attachment, stage 2 - the growth, and stage 3 - the detachment [2]. 
In stage 1 , the bacteria irreversibly attach to the surface. As the bacterial cells start to multiply, the exopolysaccharide structure is formed, and the biofilm starts making multiple towers and water channels through the matrix; these water channels play an important role regarding the waste disposal and help set the $\mathrm{pH}$. Bacteria communicate in-between inside the biofilm structure, through chemical signalling called quorum sensing, mechanism that sets the bases for the antibiotic therapy resistance.

During acute bacterial infection, antibiotic therapy can reverse symptoms, but unless the entire colonized surface is removed, the infection will reappear [3]. Infections related to bacterial biofilms are incredibly difficult to cure because of the resistance to the antimicrobial therapy.

The National Institutes of Health states that over $60 \%$ of all human bacterial affections include the presence of bacterial biofilms [4].

In patients suffering of CRS and CA, if oral antibiotic therapy is inefficient surgical therapies that include adenoid removal, endoscopic sinus surgery is recommended.

One study shows that anaerobes were cultivated from the sinuses of paediatric patients with CRS and $\mathrm{CA}$, at a rate between $2 \%$ and $100 \%$ [5], accomplished in their study a $91 \%$ success percent regarding the therapy of 22 paediatric patients diagnosed CRS during 5 weeks with antibiotic therapy with simultaneous adenoid removal surgery.

In another study made by Vandenberg and Heatley [6], was obtained the following complete simptoms resolution of CRS symptoms after adenoid removal in 25 (58\%) of 43 patients.

Other studies state that adenoid removal alone may have effects on the children that are diagnosed with CRS. All the above studies sustain that adenoid removal with or without ESS improves rhinosinusal symptoms.

Innovative thoughts concerning bacterial biofilm were generated when William G. Characklisstated stated that bacteria included in biofilm structure is resistant to the action disinfectants [7]. He postulated that bacteria do not exist alone, as planktonic but in organized systems like biofilms even in human host.

These systems of bacteria are admirably adapted for conditions of environmental stress. It has been noted that, almost $99 \%$ of the bacteria exist in biofilms, and are exceedingly difficult to eradicate [8]. During the bacterial evolution, this growth method gathers multiple advantages [9]. Several antibiotics have been tested on bacterial biofilms, but inside the biofilm, bacterial structures have proved to be much less sensitive to the treatments than the planktonic ones [10].

Bacterial biofilm on adenoid surface represents a mechanism of constant contamination for the chronic infection to the rhinosinusal mucosa [11].

To prove a new theory in the inception of CRS, we decided to display the presence biofilm on the surface of the adenoid mucosa removed from the paediatric patients diagnosed with CRS and CA.

Biofilms are communities of bacteria encapsuled in a self-generated matrix of glycocalyx and, during several studies, adenoid mases have appeared to anchor these structures [12].

In chronic infectious with adenoid hypertrophy, antibiotics therapy is appropriate to start with. Amoxicillin with a beta-lactamase inhibitor such as clavulanic acid is usually used in uncomplicated chronic adenoiditis, clindamycin or azithromycin are alternatives in allergic patients. Nasal steroids are used as additional option for medical treatment, overall, the results are unsatisfying to the efficacy of these medications [13-15].

\section{MATERIALS AND METHODS}

The study was made between January 2015 and December 2018 in the ENT department of Arad Clinical Emergencies Hospital with the approval and in compliance with the ethical principles of the ethics committee of the Hospital and of the ethic committee of the „Vasile Goldiș“" Western University of Arad, Romania, and with the Helsinki Declaration.

We subscribed in the study 50 children from the ENT department of Arad Clinical Emergencies Hospital, 28 girls and 22 boys aged between 5years and 12 years, diagnosed with CRS and CA. All the patient parents/caregivers signed a written informed consent to undergo the surgical intervention. Patients' parents/caregivers were informed regarding the surgical therapy.

All patients were submitted to full ENT (ear, nose, throat) examination, bloodwork, pulmonary radiography, allergy tests to all medication that followed to be administered, and a paediatric cardiological consult.

The examinations were documented in the medical records of the paediatric patients.

Rhinologic endoscopy was performed with the $0^{\circ}$ Storz $4 \mathrm{~mm}$ rigid endoscopes, Storz telecam SL II Camera and a Storz Halogen 250 twin light source to evidence the [presence of enlarged adenoids.

We set a definition for chronic rhinosinusitis the following: CRS as an infectious state, lasting 
longer than 3 months, that failed long term course of oral antibiotherapy for a period of minimum 5 weeks. Clinically characterized by mucopurulent rhinorrhoea, headaches, mouth breathing, irritability, facial pressure.

The definition for CA is as follows: endoscopic documented increase in the size of adenoids, snoring, mouth breathing, dry mouth, focus problems on school, fatigability, difficulty breathing during spot activities, chronic cough, post-nasal drip, sleep-disordered breathing in the absence of any allergic suspicion (sneezing, nasal itching, watery rhinorrea)

We included in the study group paediatric patients with CRS and video- endoscopic diagnosed hypertrophic adenoids (CA).

Clinically, the paediatric patients diagnosed with CRS and chronic adenoiditis experienced minimum two of the symptoms: nasal obstruction, hyposmia/anosmia, muco- purulent nasal discharge, anterior/posterior nasal drip sensation, facial fullness, facial pressure.

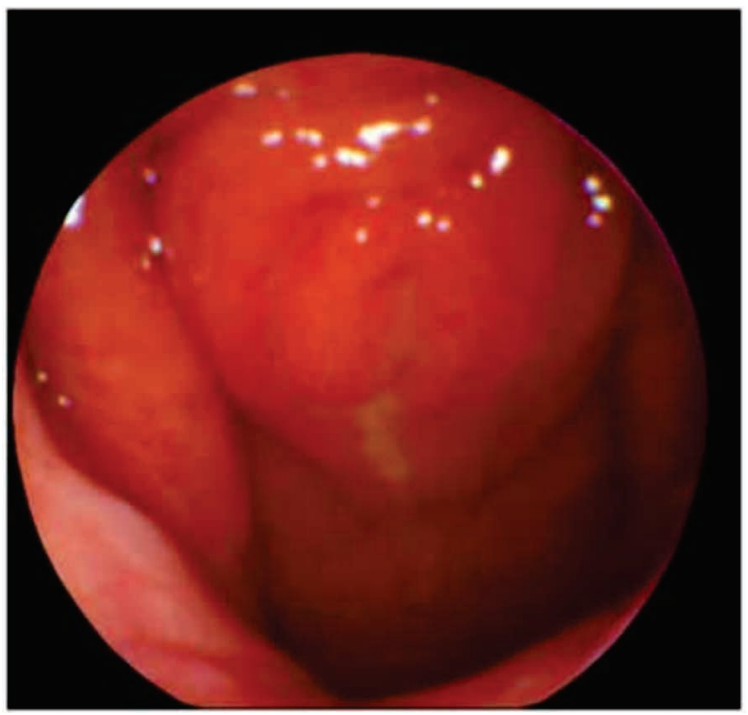

FIGURE 1. Enlarged adenoids in 7-year-old male patient- endoscopic image

There are many pharmaceutical protocols regarding the cure of CRS in paediatric patients like: antibiotics, saline lavage, topic corticosteroids. In most cases, these protocols fail to be curative.

In our clinic, on the department pf ENT od Arad Emergency Hospital we practice the following surgical protocol, concerning CRS with chronic adenoiditis: adenoidectomy, combined with endoscopic sinus surgery (ESS). All 50 patients underwent endoscopic adenoidectomy ESS.

We have removed the adenoid mases from all 50 patients included in the study and sent them for SEM preparation to our microscopy lab. The fixa- tion method for SEM adenoid specimens was realized in $2.5 \%$ glutaraldehyde in PBS buffer $(\mathrm{pH}$ 7.4) for $2 \mathrm{~h}$ at $22^{\circ} \mathrm{C}$. The pieces were post-fixed for $1 \mathrm{~h}$ with $1 \%$ osmium tetroxide in PBS at $37^{\circ} \mathrm{C}$. After fixation, the probes were dehydrated using graded ethanol series and immersed 5 times in a solution of hexamethyldisilazane for 10-15 minutes and left to dry overnight. Samples were mounted and silver sputter coated in the terminal preparation. We observed the bacterial biofilm using a scanning electron microscope FEI Quanta 250 from "Vasile Goldiș" Western University of Arad, Microscopy laboratory.

We measured the bacterial biofilm coverage percentage of the adenoid mases extracted adenoids from the 50 paediatric patients using Carnoy software.

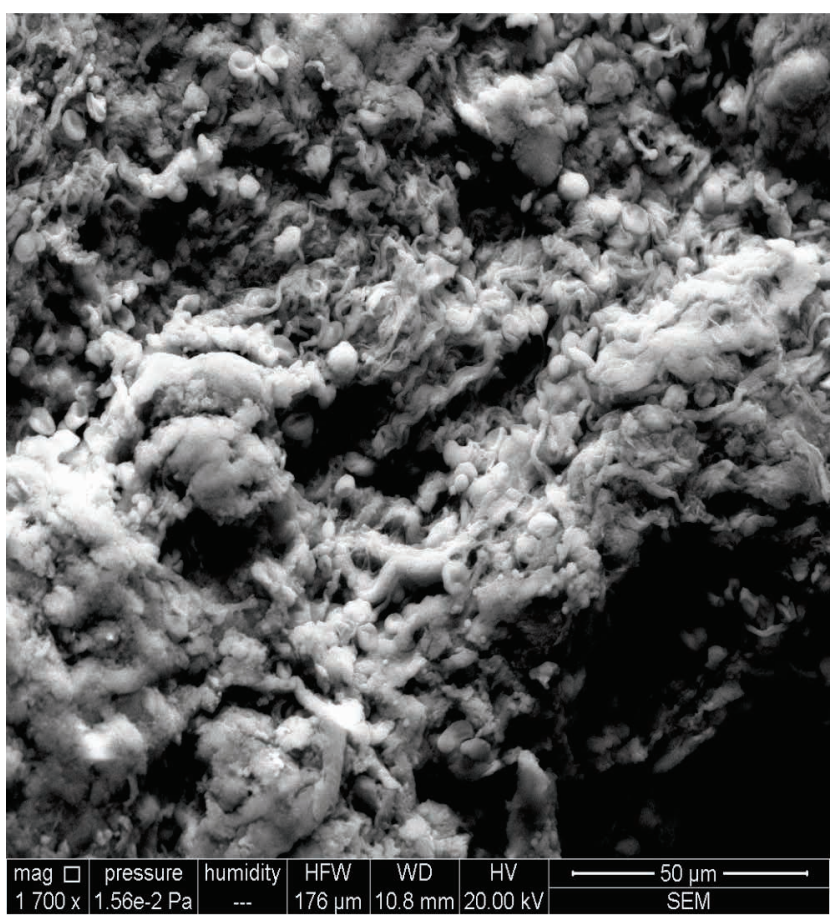

FIGURE 2. SEM image of biofilm structure on the surface of adenoid removed from CRS female 10 years old paediatric patient

In Figure 2, we can observe SEM image of adenoid tissue removed from a patient suffering from CRS.

Using Carnoy measurement software we calculated the percentage of coverage of the adenoid mucosa with bacterial biofilm of every sample examined using SEM.

\section{OUTCOMES}

Table 1 shows the obtained percentages of biofilm coverage at each of the 28 female paediatric patients, demographics, and diagnoses. 
Table 2 shows the obtained percentages of biofilm coverage at each of the 22 male paediatric patients, demographics, and diagnoses.

TABLE 1. Girls with CRS\% coverage with biofilm $84.81 \%$

\begin{tabular}{|c|c|c|}
\hline Nr. crt & $\begin{array}{c}\% \text { Coverage with } \\
\text { biofilm }\end{array}$ & age \\
\hline 1 & 79.51 & 10 \\
\hline 2 & 90.11 & 5 \\
\hline 3 & 88.72 & 7 \\
\hline 4 & 80.33 & 8 \\
\hline 5 & 77.55 & 11 \\
\hline 6 & 72.92 & 10 \\
\hline 7 & 96.64 & 6 \\
\hline 8 & 60.27 & 12 \\
\hline 9 & 93.15 & 5 \\
\hline 10 & 85.65 & 7 \\
\hline 11 & 89.38 & 5 \\
\hline 12 & 78.91 & 8 \\
\hline 13 & 93.45 & 10 \\
\hline 14 & 75.52 & 9 \\
\hline 15 & 85.41 & 7 \\
\hline 16 & 93.22 & 6 \\
\hline 17 & 90.44 & 8 \\
\hline 18 & 82.33 & 10 \\
\hline 19 & 78.72 & 11 \\
\hline 20 & 75.23 & 8 \\
\hline 21 & 90.25 & 9 \\
\hline 22 & 91.02 & 11 \\
\hline 23 & 89.01 & 8 \\
\hline 24 & 93.15 & 10 \\
\hline 25 & 99.05 & 6 \\
\hline 26 & 85.77 & 5 \\
\hline 27 & 78.98 & 6 \\
\hline 28 & 80.21 & 6 \\
\hline
\end{tabular}

TABLE 2. Boys with CRS\% coverage with biofilm $89.2 \%$

\begin{tabular}{|c|c|c|}
\hline Nr. crt & $\begin{array}{c}\text { \% Coverage with } \\
\text { biofilm }\end{array}$ & Age \\
\hline 1 & 95.34 & 5 \\
\hline 2 & 92.55 & 6 \\
\hline 3 & 88.87 & 5 \\
\hline 4 & 84.26 & 6 \\
\hline 5 & 96.85 & 9 \\
\hline 6 & 66.54 & 6 \\
\hline 7 & 89.96 & 7 \\
\hline 8 & 98.99 & 6 \\
\hline 9 & 97.12 & 6 \\
\hline 10 & 87.23 & 5 \\
\hline 11 & 74.96 & 8 \\
\hline 12 & 99.01 & 7 \\
\hline 13 & 85.21 & 8 \\
\hline 14 & 81.56 & 7 \\
\hline 15 & 92.63 & 11 \\
\hline 16 & 82.25 & 10 \\
\hline 17 & 93.22 & 9 \\
\hline 18 & 84.85 & 5 \\
\hline 19 & 96.02 & 8 \\
\hline 20 & 99.01 & 8 \\
\hline 21 & 78.99 & 7 \\
\hline 22 & 97.03 & 9 \\
\hline
\end{tabular}

Adenoids removed from all 50 patients diagnosed with CRS presented biofilms covering on $86.75 \%$ of the mucosa.

Adenoids removed from the 28 female paediatric patients with CRS presented biofilms covering on $84.81 \%$ of the mucosa. Adenoids removed from all 22 male paediatric patients diagnosed with CRS presented biofilms covering on $89.2 \%$ of the mucosa.

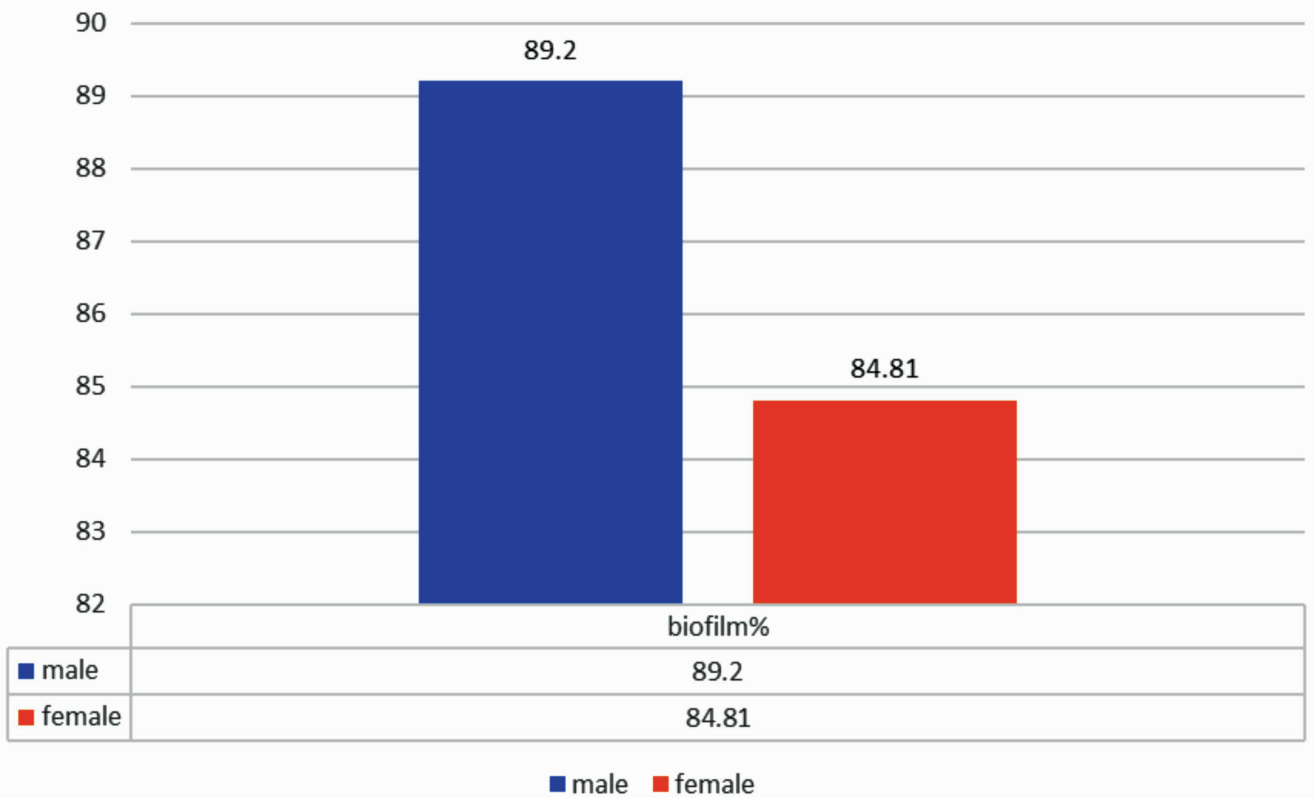

FIGURE 3. Percentage of biofilm coverage on the adenoid surface on male versus female patients 
All 50 patients had symptoms improvement.

At the 1 month control 20 patients accused intermittent nasal obstruction.

At the 3-months control 13 patients accused nasal obstruction during sleep and 2 patients accused rare episodes of headaches

At the 6-months control 2 patients accused intermittent nasal obstruction that was responds to topical saline hypertonic solutions.

\section{DISCUSSIONS}

Most of the present knowledge concerning bacterial biofilms is due to the progress in imaging research, principally in SEM.

The ability to obtain images in high magnification, with structural microscopic details, using SEM is the main advantage of the study. Even dough artifacts can arise underlying SEM processing, authors support the fact that the SEM processing does not notably modify the biofilm architecture and the recognition on samples is possible [9]. Other authors in various studies proved a prevalence of only $80 \%$ of biofilms in nasal mucosa pieces from CRS patients using SEM [10].

Bacterial biofilms were detected in $57.5 \%$ of upper respiratory mucosa by Calò et al. in a study that wants to prove that biofilm formation has a role in UA infections and that it explains the resistance of bacterial biofilms infections to antibiotic therapy [15]. The same statement is made by Galli et al. in a similar study: over $80 \%$ of the tissue specimens proved to be culture-positive and bacterial biofilm was observed in $65.6 \%$ of the tissue probes [16]. Further research made by Galli et al. proves that biofilms identified in adenoid tissue of children with recurrent inflammatory infections, may represent a bacterial "reservoir" guilty of the of the chronic inflammatory reactions, resistant to antibiotic therapy and in need of surgical treatment [17].

This study on bacterial biofilms in chronic paediatric upper inflammatory infections and on the capacity to produce biofilm in vitro, demonstrated by Haemophilus influenzae, could be related to the role of biofilms as generators in chronic infections, and to the resistance of these infections to selective antibiotic therapy [17].

The connection between biofilm and chronic infection in children is sustained by many authors, but the study of Al-Mazrou and Al-Khattaf [18] identified bacterial biofilm on the surfaces of infected hypertrophic tonsils and adenoids in most patients undergoing tonsil and adenoid removal surgery. The difference was significant regarding the presence of biofilms in children with chronic pathology, proving once again the link between the presence of biofilms and chronic infection, fact sustained by Chole and Faddis 2003 during a research that concluded that sessile bacteria in biofilms are resistant to host defences and antibiotic therapy, presence of bacterial biofilms may explain the chronicity nature of tonsillitis [19].

Our protocol is based on the debridement of the nasopharyngeal biofilm covered mucosa, and the antibiotic therapy that aims the active bacteria. Similar conclusion as the one in our study are drawn by Vlastarakos et al. and Bjarnsholt as the role of bacterial biofilms in infection becomes better defined, surgeons should be prepared to deal with their complicated nature, and research regarding adequate therapy is necessary [20,21] like strategies that include the use of particles which can dissolve the biofilm matrix and quorum sensing inhibitors, which increases biofilm susceptibility to antibiotics and phagocytosis [22-24]. After antibiotic therapy all CRS patients revealed a short-term symptom improvement, sadly followed by the fast rebound, based on the migration process of the antibiotic resistant bacteria, from the adenoids (in the rhino-pharynx) to the sinusal area [25].

The recognition that chronic ENT bacterial infections in adults and children are biofilm related has been mandatory for the development of new strategies for the examination and eradication of this type of infections [23].

Despite the limitation of the research regarding electron microscopy detection of the biofilm: risk of probe deterioration during the process of biopsy, transportation, fixation and dehydration, and the long hours spent in the microscopy laboratory room, we have observed biofilm presence in all our CRS with chronic adenoiditis patients, fact that concludes the strong point of the study.

\section{CONCLUSIONS}

Adenoids removed from the paediatric patients diagnosed with CRS behave as a source of infection, speeding up the colonization of the planktonic organisms, comforting a cycle of infection, statement is sustained by the high percentage of biofilm coverage of the adenoids, we presented at the results section $86.75 \%$.

SEM examination of the adenoid mass samples is an adequate approach of biofilm detection on adenoid tissue. 
Adenoids removed from patients diagnosed with CSR and chronic adenoiditis have most of their mucosal surface covered with biofilm.

In the nasopharynx of the patients diagnosed with CSR and CA, the bacterial biofilm has the role of a constant fountain of infection.

The CRS and CA patients had suggestive symptomatic improvement after the adenoid surgical removal at the control visits at 1, 3 and 6 months.

Antibiotic therapy protocols have short term effect, the infection reappears after periods of symptomatic peace.

Conflict of interest: none declared

Financial support: none declared

\section{REFERENCES}

1. Donlan RM. Biofilms: microbial life on surfaces. Emerg Infect Dis. 2002 Sep;8(9):881-90.

2. Ramadan HH, Sanclement JA, Thomas JG. Chronic rhinosinusitis and biofilms. Otolaryngol Head Neck Surg. 2005 Mar;132(3):414-7.

3. Costerton JW, Stewart PS, Greenberg EP. Bacterial biofilms: a common cause of persistent infections. Science. 1999 May 21;284(5418):1318-22.

4. Lewis K. Riddle of biofilm resistance. Antimicrob Agents Chemother. $2001 \mathrm{Apr} ; 45(4): 999-1007$.

5. Adappa ND, Coticchia JM. Management of refractory chronic rhinosinusitis in children. Am J Otolaryngol. 2006 Nov-Dec; 27(6):384-9.

6. Vandenberg SJ, Heatley DG. Efficacy of adenoidectomy in relieving symptoms of chronic sinusitis in children. Arch Otolaryngol Head Neck Surg. 1997 Jul;123(7):675-8.

7. Vandenberg SJ, Heatley DG. Efficacy of adenoidectomy in relieving symptoms of chronic sinusitis in children. Arch Otolaryngol Head Neck Surg. 1997 Jul;123(7):675-8.

8. Donlan RM. Biofilm formation: a clinically relevant microbiological process. Clin Infect Dis. 2001 Oct 15;33(8):1387-92.

9. Tamashiro E, Antunes MB, Palmer JN, Cohen NA, Anselmo-Lima WT. Implications of bacterial biofilms in chronic rhinosinusitis. Braz J Infect Dis. 2009 Jun;13(3):232-5.

10. Sanclement JA, Webster P, Thomas J, Ramadan HH. Bacterial biofilms in surgical specimens of patients with chronic rhinosinusitis. Laryngoscope. 2005 Apr;115(4):578-82.

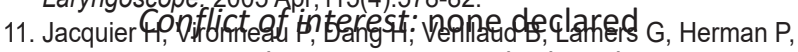

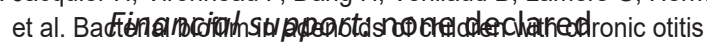
media. Part II: a case-control study of nasopharyngeal microbiota, virulence, and resistance of biofilms in adenoids. Acta Otolaryngol. 2020 Mar;140(3):220-224.

12. Kania R, Vironneau P, Dang H, Bercot B, Cambau E, et al. Bacterial biofilm in adenoids of children with chronic otitis media. Part I: a case control study of prevalence of biofilms in adenoids, risk factors and middle ear biofilms. Acta Otolaryngol. 2019 Apr;139(4):345-350.

13. Chohan A, Lal A, Chohan K, Chakravarti A, Gomber S. Systematic review and meta-analysis of randomized controlled trials on the role
Nasopharyngeal adenoid mass resection and ESS represents an appropriate approach in the therapy of the paediatric patients diagnosed with CRS and CA, nonresponsive to the antibiotic treatment.

The study has a high practical significance especially for the paediatric practicians and family doctors that fight day by day with paediatric upper airway pathology, setting a clear therapeutical axioma: Patents diagnosed with CRS and chronic adenoiditis nonresponsive to antibiotic therapy for more than 5 weeks should consider ESS. of mometasone in adenoid hypertrophy in children. Int $\mathrm{J}$ Pediatr Otorhinolaryngol. 2015 Oct;79(10):1599-608.

14. Demirhan H, Aksoy F, Ozturan O, Yildirim YS, Veyseller B. Medical treatment of adenoid hypertrophy with "fluticasone propionate nasal drops". Int J Pediatr Otorhinolaryngol. 2010 Jul;74(7):773-6.

15. Calò L, Passàli GC, Galli J, Fadda G, Paludetti G. Role of biofilms in chronic inflammatory diseases of the upper airways. $A d v$ Otorhinolaryngol. 2011; 72:93-6.

16. Galli J, Ardito F, Calò L, Mancinelli L, Imperiali M, et al. Recurrent upper airway infections and bacterial biofilms. J Laryngol Otol. 2007 Apr;121(4):341-4.

17. Galli J, Calò L, Ardito F, Imperiali M, Bassotti E, Fadda G, Paludetti G. Biofilm formation by Haemophilus influenzae isolated from adeno-tonsil tissue samples, and its role in recurrent adenotonsillitis. Acta Otorhinolaryngol Ital. 2007 Jun;27(3):134-8.

18. Al-Mazrou KA, Al-Khattaf AS. Adherent biofilms in adenotonsillar diseases in children. Arch Otolaryngol Head Neck Surg. 2008 Jan;134(1):20-3.

19. Chole RA, Faddis BT. Anatomical evidence of microbial biofilms in tonsillar tissues: a possible mechanism to explain chronicity. Arch Otolaryngol Head Neck Surg. 2003 Jun;129(6):634-6.

20. Vlastarakos PV, Nikolopoulos TP, Maragoudakis P, Tzagaroulakis A, Ferekidis E. Biofilms in ear, nose, and throat infections: how important are they? Laryngoscope. 2007 Apr;117(4):668-73.

21. Bjarnsholt $\mathrm{T}$. The role of bacterial biofilms in chronic infections. APMIS Suppl. 2013 May;(136):1-51.

22. Nistico L, Kreft R, Gieseke A, Coticchia JM, Burrows A, Khampang P, Liu Y, et al. Adenoid reservoir for pathogenic biofilm bacteria. J Clin Microbiol. 2011 Apr;49(4):1411-20.

23. Post JC, Hiller NL, Nistico L, Stoodley P, Ehrlich GD. The role of biofilms in otolaryngologic infections: update 2007. Curr Opin Otolaryngol Head Neck Surg. 2007 Oct;15(5):347-51.

24. Høiby N, Ciofu O, Johansen HK, Song ZJ, Moser C, et al. The clinical impact of bacterial biofilms. Int J Oral Sci. 2011 Apr;3(2):55-65.

25. Høiby N, Bjarnsholt T, Givskov M, Molin S, Ciofu O. Antibiotic resistance of bacterial biofilms. Int J Antimicrob Agents. 2010 Apr;35(4):322-32. 